\title{
PROMOTING SELF-REGULATED LEARNING STRATEGIES FOR FIRST-YEAR STUDENTS THROUGH THE COMPER SERVICE
}

\author{
Laëtitia Pierrot ${ }^{1}$, Christine Michel ${ }^{1}$, Julien Broisin ${ }^{2}$, Nathalie Guin ${ }^{3}$, Marie Lefevre ${ }^{3}$ \\ and Rémi Venant ${ }^{4}$ \\ ${ }^{1}$ Université de Poitiers, TECHNE EA 6316, F-86073 Poitiers, France \\ ${ }^{2}$ Université Toulouse 3 Paul Sabatier, IRIT, UMR 5505, F-31062 Toulouse, France \\ ${ }^{3}$ Université de Lyon, CNRS, LIRIS UMR 5205, F-69622 Villeurbanne, France \\ ${ }^{4}$ Le Mans Université, LIUM EA 4023, F-72085 Le Mans, France
}

\begin{abstract}
Implementing remote and blended higher education courses motivated the design for new support services for autonomous learning. Thus, combining a competence-based approach and self-regulation, the COMPER project offers a service to be used in addition to the courses. It consists of a graphical presentation of the learners' competency profile (following the open learner model's approach) and a personalized resources recommendation system. To assess its usefulness and usability, we conducted a study to test the COMPER service on 181 first-year students (from a two-year university diploma in computing), in addition to practical work carried out remotely. Based on a survey (usage data and scales) analysis, our study shows that globally the learners perceive the service as useful, especially those who favor working individually. Finally, our findings showed how much attention must be paid before implementing an independent OLM into an existing learning environment, especially for learners lacking SRL competencies.
\end{abstract}

\section{KEYWORDS}

Self-Regulated Learning, Usability, Utility, Autonomous Learning, Competency-Based Approach

\section{INTRODUCTION}

One of the many students' challenges relates to the competencies they need for organizing or achieving work. However, most students entering the university have no training in the implementation of these methods. Indeed, a study on prospective students' habits (Panadero et al., 2020) shows that the most common learning strategy (70.7\% of the sample) is the organization and transformation of instructional content through creating summaries or concept maps. Marginal strategies are information seeking $(6.7 \%)$, goal setting and planning $(4 \%)$, or asking teachers for help (4\%). Only one participant reported seeking help from peers. Other strategies (self-assessment, monitoring, environmental structuring) are non-existent. Thus, there is a need to provide learners with support for autonomy in their learning activities. This problem is even more critical considering that most of these activities shifted to emergency remote mode with the health crisis.

To address this problem, helping students self-regulate (Zimmerman, 2000) and competency-based approaches (CBA) are two of the many existing strategies. Schunk (1994) defines self-regulation of learning as "a set of processes by which subjects activate and maintain goal-directed cognitions systematically, affects, and behaviors."

Our study measured the usability and relevance of combining two tools, available during face-to-face and distance courses, and designed to support students' self-regulation strategies based on CBA: access to a self-training platform and the visualization of a competency profile. We observed the use and opinions of 181 first-year students from a two-year university diploma in computing engaged in an introductory Shell programming course that gave access to these tools. We cross-referenced these with their self-regulated learning (SRL) profiles. This paper presents our findings and recommendations for designing and implementing services articulating self-regulation and CBA. 


\section{SUPPORTING STUDENTS' AUTONOMOUS WORK AT THE UNIVERSITY}

Self-regulation skills have been found as a factor for learners' performance. Using these skills allows them to efficiently control and modify their actions to reach set goals (Pintrich, 2000). Instead of simply responding to environmental stimuli, learners organize their environment according to their targets. This approach includes different strategies that the learners can implement: self-assessment, studying training resources, asking for help or defining objectives, and planning their work, among many (Zimmerman and Pons, 1986).

\subsection{Promoting SRL Strategies}

The tools proposed to measure and promote self-regulation have evolved in three waves (Panadero, 2017). The first employed learners' self-reported data from questionnaires, the second trace data from learners' interactions with learning platforms. In both cases, the data's exploitation provided learners (or teachers) with global pictures useful for making diagnoses and adapting their behaviors. The third wave of tools also exploits interaction trace data and adds functionalities that stimulate learners' self-regulation skills. The most common functionalities in current devices (2008-2018) correspond to tools from waves 2 and 3 and include dashboards fed from Learning Analytics (LA) techniques, intelligent agents, and personalized feedback/prompts offered to learners. However, studies (Araka et al., 2020; Panadero, 2017) have pointed out the need to exploit further LA and the lack of models to implement third wave tools.

Schumacher and Ifenthaler (2018) analyzed how different functionalities of interest for SRL can proceed from interaction data. In parallel, they evaluated their usefulness and acceptability to learners. Their study showed that the most useful feature is the invitation to self-evaluate with immediate feedback to support the reflection and performance phases. Indeed, this feature is necessary to assess the state of knowledge and plan the next steps. Furthermore, the following two most useful features are the recommendation of topics and courses to work on based on, for example, the content the learner has already worked on. Finally, the features more oriented towards planning or monitoring the activity are not considered useful or acceptable.

Self-assessment with immediate feedback requires activities or features that allow students to assess themselves against the course objectives. Exercisers (or exercise generators) are a way to diagnose the skills acquired quickly, to perform performance memorization and skill development through trial and error learning based on repetition (Mostow et al., 2004). Since the exerciser environment keeps track of the learning activity and provides immediate feedback, it facilitates the learner's regulation by allowing explicit reflection on the skills worked on (Steffens, 2006). The following section presents specific visualizations of the skills profile useful to help the learners situate themselves under the competency framework.

\subsection{Competency Profile Visualization}

Dashboards include various information to visualize the skills evolution according to the success in learning activities, especially for an online or hybrid learning context (Kizilcec et al., 2017). Visualizing objectives provides learners with standards against which to position themselves (Sedrakyan et al., 2019). However, these strategies have limitations in terms of effectiveness. Only older learners have the metacognitive skills to implement SRL processes (Kizilcec et al., 2017). Moreover, these strategies must be combined with assessments so that learners can verify the achievement of objectives. Finally, statistical approaches, which are currently widely applied, should be complemented with techniques that incorporate the level of readiness and the dependencies between learning objectives and sub-objectives (Sedrakyan et al., 2019). This type of formalization particularly occurs in CBA.

The OLM is a structured representation of the state of one's correct/incorrect knowledge and difficulties. It allows the learner (and/or others) to visualize information about themselves (Bull and Kay, 2010), according to an awareness process that supports learning (Bodily et al., 2018). Bull and Kay (2010) identified several benefits for the learner, with the OLM supporting metacognitive activities, facilitating consultation and access to related resources, and making the learner more autonomous as they are more responsible for their learning. However, OLMs are generally dependent on a learning device, making them unusable for others (Hooshyar et al., 2020). The simplest forms using color coding are preferred as learners more easily use them. Complex 
representations, which hierarchically show knowledge structures, are more useful for supporting SRL processes but are also the most difficult to use (Hooshyar et al., 2020). It is, therefore, a challenge to find a good visual representation for an independent OLM. This literature review also shows the difficulties of knowing which visual representation a learner would prefer depending on SRL skills.

In this paper and in the COMPER project, we aim to study different visualization forms of independent OLMs and measure their usefulness and usability according to the SRL skills of the learners.

\section{SUPPORTING SELF-REGULATION IN AN INTRODUCTORY SHELL PROGRAMMING COURSE}

\subsection{Learning Situation Experimented}

The COMPER project produced several independent tools to support self-regulation: a competency framework, a personalized resource recommendation system, and a tool for visualizing learner competency profiles (OLM module). Combining these tools allows us to cover the three stages of the SRL cycle.

The OLM module proposed a description of the learner's knowledge, skills, and competency mastery rate, according to the learning objectives defined within the framework. The mastery rate was calculated according to the learner's success in the learning activities, based on the data-trace analysis (if a remote system is used) and/or teachers' opinions. The OLM module also provided a confidence rate to indicate the mastery rates' reliability based on the number of assessments and the assessments' recency. According to previous studies within the COMPER project (Bull et al., 2013; Hooshyar et al., 2020), four different profile visualizations were designed (see Figure 1).

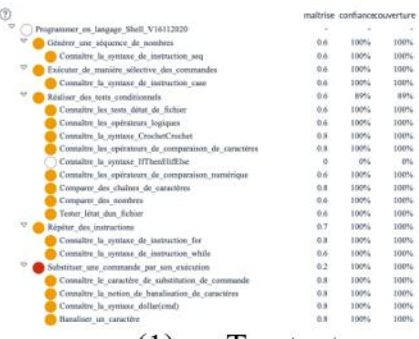

(1) Treetext

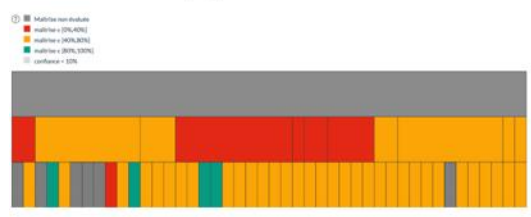

(3) Treemap

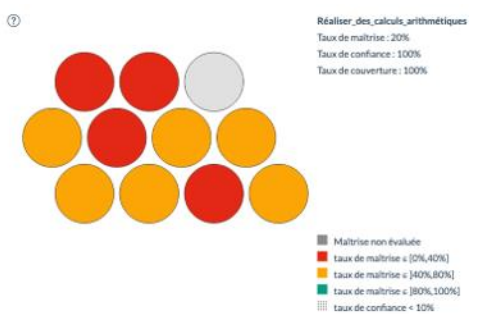

(2) Treepack

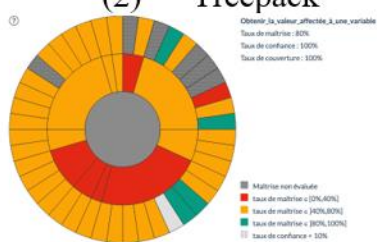

(4) Sunburst

Figure 1. OLM module visualizations in the COMPER project

We experimented with a combination of tools for an introductory Shell programming course offered to first-year university students starting November 2020. A virtual and remote laboratory (Broisin et al., 2017) is usually operated in this course. It provides learners with an infrastructure of virtual machines and networks adapted to the course's needs. We add three tools: a competency framework, the OLM module, and an exercise generator (exerciser platform) (Lefevre et al., 2015) used for several years in university but never in this course. The competency framework represented the 55 competencies "Programming in Shell language" worked in the class. We propose access to the OLM module through the virtual and remote laboratory's interface because this tool is usually used. Finally, the teacher provided a set of exercises for individual training with the exerciser platform.

We implemented this combination to correspond to the learners' preferences (Schumacher and Ifenthaler, 2018) and respect the design recommendations identified previously (Sedrakyan et al., 2019). The exerciser platform (Lefevre et al., 2015) allows teachers to create self-evaluation exercises and provides automatic and 
instant evaluation, so learners receive immediate feedback. OLM module helps learners to visualize their competency profiles and to supervise knowledge and skills.

\subsection{Research Questions}

In addition to verifying the feasibility of combining these different tools in an existing training course, this implementation in the programming course aimed to answer several research questions. RQ1: Globally, how useful is the whole combination in terms of self-regulation? More specifically (RQ1.1), is the support useful for all students or just for those who lack SRL strategies? RQ2: Are the tools usable by first-year university students who work autonomously overall and have punctual guidance? More specifically (RQ2.1), are the visualizations proposed in the competency profile easy to understand and use by students?

\section{STUDY}

\subsection{Participants}

181 participants took part in this experiment. All of them were students enrolled in the first year of a two-year diploma in computing (French Diplôme Universitaire de Technologie), aged between 17 and 19 years old, mostly male ( $87 \%$ male to $13 \%$ female). The students followed the training as described above, working alone and remotely.

\subsection{Shell Programming Course Organization}

Traditionally, this course is on a face-to-face modality, and it was conducted remotely due to the sanitary crisis. Learners had access to the tools during the five remote practical work sessions (once a week), followed by three weeks of revision and a face-to-face exam. Before the course, the teacher defined the course's objectives within the competence framework, the practice exercises within the virtual and remote laboratory, and those carried out with the exerciser platform.

During the practical sessions, the teacher attended learners remotely to complete the practical work and corrected their production at the end of each week. He assessed the learners' skills, calculating the competency profile and updating the framework system elements' mastery rates. Between sessions, learners had access to a list of resources adapted to their competency profile. Resources included course materials and exercises from the exerciser platform. Learners could freely follow the system's recommendations or autonomously choose exercises. All the tools were available during the training phase (during the five weeks of practical work courses) and the revision phase.

To evaluate each profile visualization, all participants were divided, at the beginning of our study, into four experimental groups (45 to 46 students per group) in a balanced way according to their academic level. During the first two weeks of the training phase, each group had only access to a single profile visualization among the four available. After these two weeks, each learner could freely access all four visualizations and switch to the preferred one at any moment.

\subsection{Collected Data}

In this study, we carried out several measurements through online surveys. First, the EAREL scale (Cosnefroy et al., 2020), which focuses on SRL strategies for distance learning activities, measured learners' SRL skills. Students were asked to indicate their disagreement (1: strongly disagree) or agreement (7: strongly agree) on 4 dimensions of 6 items each, namely procrastination (PROC), control of the learning context (CTXT), cognitive/metacognitive strategies used for learning (COGN), and peer support (PEERS). We used this scale at the beginning (EAREL, $\mathrm{n}=94$ ) of the experiment to cover RQ1.

According to the System Usability Scale, the second scale evaluates the OLM module's usability (Brooke, 1996). The SUS measures usability through 10 items, consistent with an agreement scale (1: strongly disagree, 5: strongly agree). The score obtained is displayed out of 100. A system's usability is considered correct, but 
with usability problems when the SUS is between 50 and 70, good when it is between 71 and 85 , and excellent if it is above 85. We used this scale to cover RQ2 (SUS, $n=73$ ). To balance self-reported data on usability, we used logs from the exerciser platform $(n=98)$ and OLM module $(n=82)$. Each log contains data on the learners' activity (temporally marked interactions with the system).

Besides, we remotely conducted semi-structured interviews with 5 students at the end of the experiment. These interviews aimed to deepen their understanding of the tools, their work strategies, and their wishes for the tools' evolution. In this paper, we use the quotes of 5 students to illustrate and complete the analyses.

\section{RESULTS}

\subsection{Learners' SRL Competencies}

According to EAREL scale results, learners show good abilities to organize their learning context. However, they have a high propensity to procrastinate and demonstrate poor communication and work strategies. In addition, the standard deviations and coefficients of variation are high, which indicates a strong population disparity (see Table 1).

Table 1. Mean (m), standard deviation $(\sigma)$, and coefficient of variation $(\mathrm{CV})$ on EAREL dimensions

\begin{tabular}{lllll}
\hline SRL strategies & CTXT & PEERS & PROC & STRAT \\
\hline m before $(\mathrm{n}=94)$ & 4,84 & 4,13 & 4,07 & 3,24 \\
$\sigma$ before & 1,00 & 1,56 & 1,40 & 1,33 \\
CV before & $21 \%$ & $38 \%$ & $34 \%$ & $41 \%$ \\
\hline
\end{tabular}

We performed a principal component analysis (PCA) and a K-means partition to view various SRL profiles globally. We applied PCA to explore linear relationships among the 24 variables. We found 3 characterizing variables: procrastinating and implementing work strategies (with the highest values on the left side of Axis 1 in Figure 2) and communicating with peers (with the highest values on the top of Axis 2). Contextual control behaviors were not discriminating. The K-means method allowed us to identify four groups of learners structured according to their implementation level of SRL strategies. Figure 2 shows learners' distribution according to the K-means groups in the vector plane composed of PCA axes 1 and 2. Dropouts (class1-blue) do not use any strategy, procrastinate and communicate little with their peers. Followers (class2-yellow) use some strategies but procrastinate and start working by communicating with their peers. Lone enforcers (class3-green) use strategies, do not procrastinate and do not communicate with their peers. Finally, efficient students (class4-orange) use strategies, do not procrastinate and communicate with their peers.

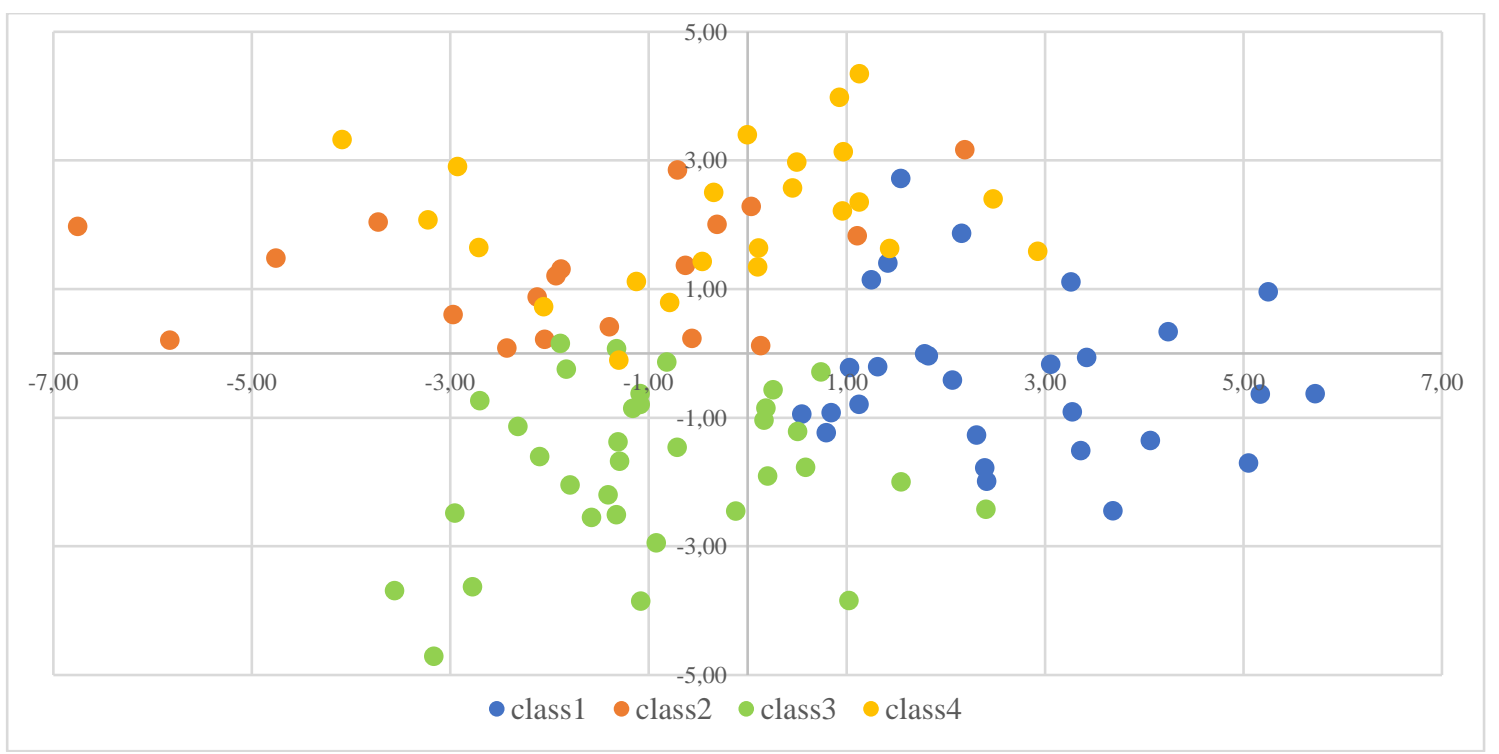

Figure 2. Learners distribution by SRL characteristics (level of procrastination, strategy use, and request for peer support) 


\subsection{Analysis of COMPER Project's Combination Use and Usefulness}

\subsubsection{Analysis of the Exerciser Platform Use and Usefulness}

According to the exerciser platform's log analysis (cf. figure 3a), half of the students did not use it (83/181), mostly because they lacked time. However, $83.56 \%$ of the learners who declared using it found it useful (see figure $3 \mathrm{~b}$ ). Most of the learners who used the exerciser platform are the least communicative: class 1 (dropouts) and class3 (lone-enforcers). "I practice until I get it. I do sequential work" self analyzes a class1-student while a class3-student mostly acts out of a performance quest: “if I really can't do it, I'll get help, but otherwise I try to succeed on my own". This use was motivated by the students' desire to position themselves in their learning process (to check if they have understood and know where they stand) and target and work on specific skills. "I try to follow and learn in class so that I can limit the amount of work I have to do at home, and for the exams, it's the same" explains a class4-student.

\subsubsection{Analysis of the OLM Module Use and Usefulness}

The OLM module log analysis showed that an even larger proportion of students did not use the OLM module (102/181, see figure 4a). The OLM module was considered not useful (at $45.83 \%$ ) and difficult to use (at $55.55 \%$ ) (see figure $4 \mathrm{~b}$ ). Also, some students reported being unaware of the service and relying on other resources, such as direct feedback from the instructor, to assess their progress. A class1-student reported: “Actually, I think I didn't understand how to use the competency profile because I didn't see it changing much, so I stopped looking at it". The other class1-student found the OLM module adequate to practical works, but he explained not using it since he didn't like "having to prove" what he learned. When used, it was mostly at a low level (see Figure 4a). A class2-student explained how he used the OLM module after the course: "I would look to see if it turned green. It reassured me to know that the system said I understood". Learners used the tool to monitor their overall progress on the course, knowing that the teachers' previous week's assessment was added into the competency profile.

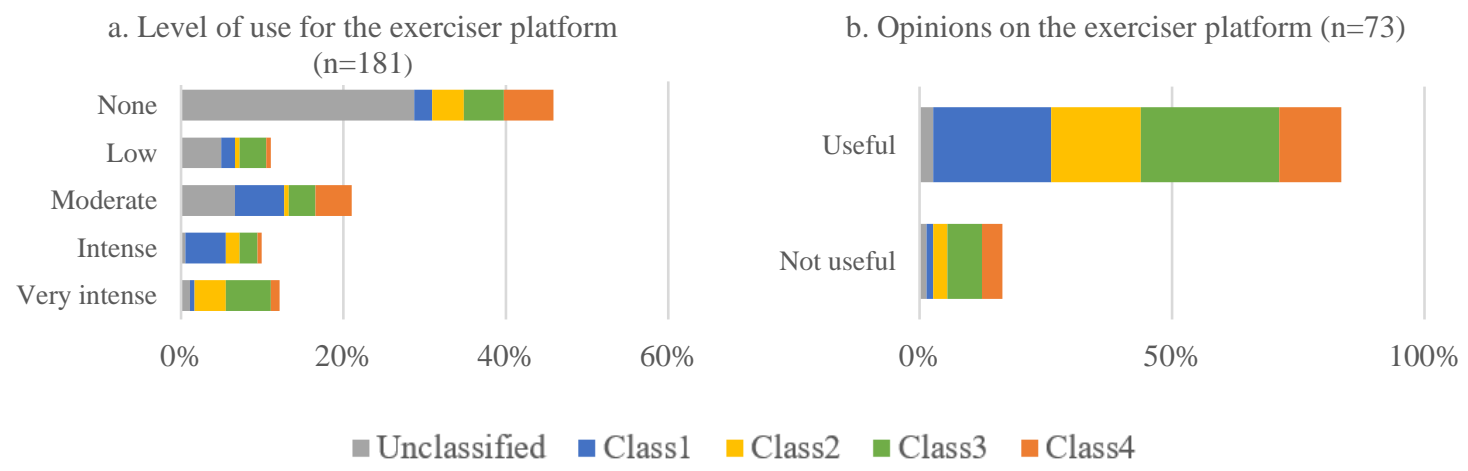

Figure 3. Level (a), and students' opinion (b) for the exerciser platform. Unclassified group shows logs activity from students who did not complete the EAREL scale

a. Level of use for the OLM module $(n=181)$

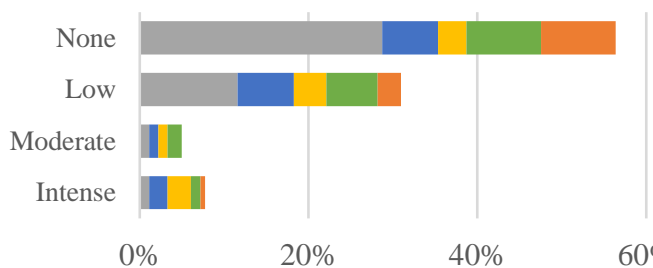

b. Opinions on the OLM module ( $\mathrm{n}=73$ )

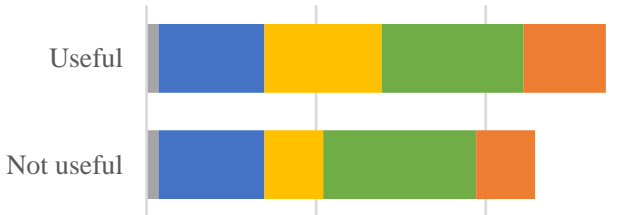

$0 \% \quad 20 \% \quad 40 \%$

$60 \%$

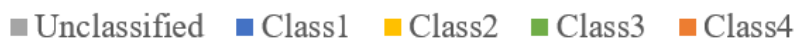

Figure 4. Level (a), and students' opinion (b) for the OLM module 


\subsection{Opinions about the OLM Module Design}

\subsubsection{OLM Module Usability}

If the exerciser platform was found easy to use (49 out of 73 learners who declared using it), only half of students found the OLM module easy to use (32 out of 64 learners who declared using it). Indeed, the OLM module obtained a SUS score of 59.49/100. The OLM module has, therefore, correct but limited usability. These limitations are the need for technical support (average score of 3.167/4) and difficulties in using OLM (items "I thought there was too much inconsistency in this system" average score of 3.056/4, and "I find this service very cumbersome to use" average score of 3.022/4). However, the analysis of the trace data activity on the OLM module showed that only a minority of the logs concern technical errors encountered by the students, either when displaying a skill or changing the visualization. The type of difficulties encountered is thus more related to the type of visualization. On the other hand, students expressed feeling unconfident with the information displayed (average score of 1.8/4) and did not find the service well integrated (average score of 2/4). Another explanation may be related to a misunderstanding of some SUS items.

\subsubsection{Comparison of the Four Visualizations}

According to the activity logs, the students consulted the Treetext visualization $(46 \%)$. When learners chose the four visualizations, they systematically replaced the sunburst visualization with another, mainly the Treetext (52\%). The opinions expressed in the questionnaire confirm this and show that the preferred view is the Treetext (for 37 learners out of 47). For them, this visualization is easy to read and understand, and it allows them to grasp quickly each targeted skill, which was confirmed by four of the five students interviewed. On the other hand, 5 out of 47 learners preferred the Treemap presentation. Like with the Treetext visualization, the Treemap offered "clear" and "comprehensive" information. However, one student expressed his concerns about the other two visualizations, mentioning how they were not enough "intuitive and too esthetic." In contrast, another explained how Treetext and Treemap visualizations were less complex for them. A few students (5 out of 47) preferred the two other visualizations (conversely, 3 for the Sunburst visualization, 2 for the Treepack one). In that case, these visualizations were the first ones they have had access to.

\section{DISCUSSION}

The students' SRL profile played an important role in the use of the tools. "Dropout" students, characterized by a lack of SRL strategies, mainly used reinforcement exercises, but the OLM module was not yet usable for them. The "followers" and "efficient" students were less likely to use the COMPER service. The "effective" ones, already using SRL strategies, consider these tools not very useful for additional help. The "followers" mostly used profile visualization during practical work courses, which suggests that it was at the teacher's request. Thus, the COMPER service appears to address the needs of some students who are isolated and have little or no SRL strategies in place. It would be interesting to compare the learners' goals (mastery or performance) to see if, as for Lust et al. (2013) or Matcha et al. (2019), they guide the preference of use towards monitoring tools (such as the OLM module) or testable materials (such as the exerciser platform).

The exerciser platform was found to be more usable than the OLM module, considered difficult to understand. However, its use, which did not stop, and the statement about the Treetext visualization, judged easy to read and understand, invites further development. Indeed, the results confirm the link between perceived usability and the tools' actual usefulness: the students who most favorably evaluated the tools were also those who used them the most and for the longest time. Further research is needed to understand what design features lead learners to believe that this visualization is easier to use.

In this study, although the OLM module was implemented directly in the learning environment that students were used to, it was conceived as an independent OLM. This choice was made since the overall COMPER project aims to design and implement generic tools to existing learning situations. Yet, our usability findings showed that the module was not perceived as part of their usual environment for some learners. Still, Hooshyar et al. (2020) recommend maintaining the OLM module independent or open to various resources or materials. Lust et al. (2013) showed that much attention needs to be provided before students start using a new SRL tactic. Thus, the scaffolding (offered by the teacher at the beginning of the experiment and by the system through the 
profile captions) may not have been sufficient for learners to adopt the tools fully. The analysis of the scaffolding means seems to be a good research perspective to improve the design and implementation of such services. The editable aspect of the exerciser platform, which was much more used than the OLM module, invites us to consider adding more personalized/inspectable features to further development of the OLM module.

\section{CONCLUSION}

This article describes the COMPER service's experimentation by first-year students in computing, articulating a competency-based approach and self-regulation to support autonomous work. The experimentation showed that both tools (exerciser platform and OLM module) were globally useful and usable, but in different ways according to the learners' SRL profiles. The tools mainly address the learners' needs who have not developed SRL strategies. The research also shows that the other SRL profiles used the set of tools less. Exploiting the data from the questionnaires and the activity logs confirmed the global approach's relevance with students in autonomous learning situations. Our future work will focus on better understanding learners' motivations for using these services and how best to adapt the design and implementation according to their needs and the characteristics of learning sessions.

\section{ACKNOWLEDGEMENT}

This experimentation and the design of the tools were carried out in the context of the French National Research Agency COMPER project (ANR-18-CE38-0012).

\section{REFERENCES}

Araka, E., Maina, E., Gitonga, R., Oboko, R., 2020. Research trends in measurement and intervention tools for self-regulated learning for e-learning environments - systematic review (2008-2018). RPTEL 15, 6.

Bodily, R., Kay, J., Aleven, V., Jivet, I., Davis, D., Xhakaj, F., Verbert, K., 2018. Open learner models and learning analytics dashboards: a systematic review. pp. 41-50. https://doi.org/10.1145/3170358.3170409

Broisin, J., Venant, R., Vidal, P., 2017. Awareness and Reflection in Virtual and Remote Laboratories: the case of Computer Education. International Journal of Technology Enhanced Learning 9, 1.

Bull, S., Kay, J., 2010. Open Learner Models, in: Nkambou, R., Bourdeau, J., Mizoguchi, R. (Eds.), Advances in Intelligent Tutoring Systems, Studies in Computational Intelligence. Springer Berlin Heidelberg, Berlin, Heidelberg, pp. 301-322. https://doi.org/10.1007/978-3-642-14363-2_15

Bull, S., Kickmeier, M., Vatrapu, R., Johnson, M., Hammermueller, K., Byrne, W., Hernandez-Munoz, L., Giorgini, F., Meissl-Egghart, G., 2013. Learning, Learning Analytics, Activity Visualisation and Open Learner Model: Confusing? https://doi.org/10.1007/978-3-642-40814-4_51

Cosnefroy, L., Fenouillet, F., Heutte, J., 2020. Construction et validation de l'Échelle d'autorégulation des apprentissages en ligne (EAREL). Canadian Journal of Behavioural Science / Revue canadienne des sciences du comportement 52, 255-260.

Hooshyar, D., Pedaste, M., Saks, K., Leijen, Ä., Bardone, E., Wang, M., 2020. Open learner models in supporting self-regulated learning in higher education: A systematic literature review. Computers \& Education 154, 103878. https://doi.org/10.1016/j.compedu.2020.103878

Kizilcec, R.F., Pérez-Sanagustín, M., Maldonado, J.J., 2017. Self-regulated learning strategies predict learner behavior and goal attainment in Massive Open Online Courses. Computers \& Education 104, 18-33.

Lefevre, M., Guin, N., Cablé, B., Buffa, B., 2015. ASKER : un outil auteur pour la création d'exercices d'auto-évaluation, in: Atelier EAEI (Évaluation Des Apprentissages et Environnements Informatiques) - Conférence EIAH 2015. Agadir, Morocco.

Lust, G., Elen, J., Clarebout, G., 2013. Students' tool-use within a web enhanced course: Explanatory mechanisms of students' tool-use pattern. Computers in Human Behavior 29, 2013-2021. https://doi.org/10.1016/j.chb.2013.03.014 
Matcha, W., Ahmad Uzir, N., Gasevic, D., Pardo, A., 2019. A Systematic Review of Empirical Studies on Learning Analytics Dashboards: A Self-Regulated Learning Perspective. IEEE Transactions on Learning Technologies PP, 1-1. https://doi.org/10.1109/TLT.2019.2916802

Mostow, J., Beck, J., Beyi, J., Cuneo, A., Sison, J., Tobin, B., Valeri, J., 2004. Using automated questions to assess reading comprehension, vocabulary, and effects of tutorial interventions. Tech. Inst. Cognition and Learning 2, 103-140.

Panadero, E., 2017. A Review of Self-regulated Learning: Six Models and Four Directions for Research. Front. Psychol. 8,422 .

Panadero, E., García-Pérez, D., Fernández-Ruiz, J., Sánchez-Centeno, H., 2020. A Transitional Year Level to Higher Education: Challenges, Experiences and Self-regulatory Strategies during the Final Year of the University Preparatory Level. Estudios sobre Educación 39, 109-133.

Pintrich, P.R., 2000. The role of goal orientation in self-regulated learning, in: Handbook of Self Regulation. Academie Press, San Diego, pp. 451-502.

Schumacher, C., Ifenthaler, D., 2018. Features students really expect from learning analytics. Computers in Human Behavior 78, 397-407.

Schunk, D., 1994. Self-regulation of self-efficacy and attributions in academic settings, in: Self-Regulation of Learning and Performance: Issues and Educational Applications. Hillsdale, NJ: Lawrence Erlbaum, pp. 75-99.

Sedrakyan, G., Mannens, E., Verbert, K., 2019. Guiding the choice of learning dashboard visualizations: Linking dashboard design and data visualization concepts. Journal of Computer Languages 50, 19-38.

Steffens, K., 2006. Self-Regulated Learning in Technology-Enhanced Learning Environments: lessons of a European peer review. European Journal of Education 41, 353-379.

Zimmerman, B.J., 2000. Attaining self regulation. A social cognitive perspective, in: Handbook of Self Regulation. Academie Press, San Diego, pp. 13-39.

Zimmerman, B.J., Pons, M.M., 1986. Development of a Structured Interview for Assessing Student Use of Self-Regulated Learning Strategies. American Educational Research Journal 23, 614-628. 